\title{
Systemic juvenile idiopathic arthritis with skin eruption and dactylitis
}

\section{Takako Miura, Toshiyuki Yamamoto}

\author{
Department of Dermatology, Fukushima Medical University, Fukushima, Japan
}

Corresponding author: Prof. Toshiyuki Yamamoto, E-mail: toyamade@fmu.ac.jp

Sir,

Still's disease was initially reported as a childhood disease. Adult-onset Still's disease (AOSD) and systemic juvenile idiopathic arthritis (sJIA) exist on a spectrum, and it has been generally considered that some cases occurring before age 16 years are sJIA while cases presenting after age 18 years are AOSD [1]. However, there are indeterminate cases in which sJIA is identical to adolescent-onset Still's disease. The skin rashes of both disorders are similar, and transient, salmon-pink, macular or urticarial erythema appear on the face, trunk and extremities, along with fever spikes. We herein report an indeterminate case of a patient who developed salmon-pink rashes and dactylitis.

A 17-year-old boy visited the Rheumatology department of our hospital, complaining of spike fever up to $39^{\circ} \mathrm{C}$, joint pain of the elbows and knees, and asymptomatic erythemas on the extremities that had appeared over the previous six months. During hospitalization for detailed examination, the patient was referred to our department for further examination of his skin manifestations. Erythemas appeared in parallel with fever-up, and spontaneously disappeared when his fever was reduced. On physical examination, scattered salmon-pink erythemas were observed on the bilateral feet (Fig. la). Moreover, diffuse swelling of the fingers was observed bilaterally (Fig. lb). Laboratory examination showed leukocytosis $(6,000 /$ $\mu \mathrm{l})$, elevated C-reactive protein $(6.22 \mathrm{mg} / \mathrm{dl})$, elevated antistreptolysin antibody $(976 \mathrm{mg} / \mathrm{dl}$; normal<240), normal liver function, and normal ferritin levels (190 ng/ml; normal: 50-200). Serological tests for rheumatoid factor, antinuclear antibody, anti-DNA antibody, and anti-Sm antibody were all within normal ranges. Tests for acute infection for Epstein-Barr virus, herpesvirus, and cytomegalovirus were all negative. Examination by Gallium scintigraphy revealed increased uptake on the shoulders, knees, and ankles. Two biopsies were taken, one from the dorsum and one from the sole of the foot, both of which revealed similar findings, such as normal epidermis, and infiltration of mononuclear cells and neutrophils in the dermis and subcutis (Figs. 2 and 3). Direct immunofluorescence showed negative deposition of immunoglobulins and $\mathrm{C} 3$ in the vessel walls. The patient was treated with oral prednisolone, methylprednisolone pulse $(1000 \mathrm{mg} /$ day for three consecutive days), methotrexate, tocilizumab, and infliximab at the Rheumatology department.

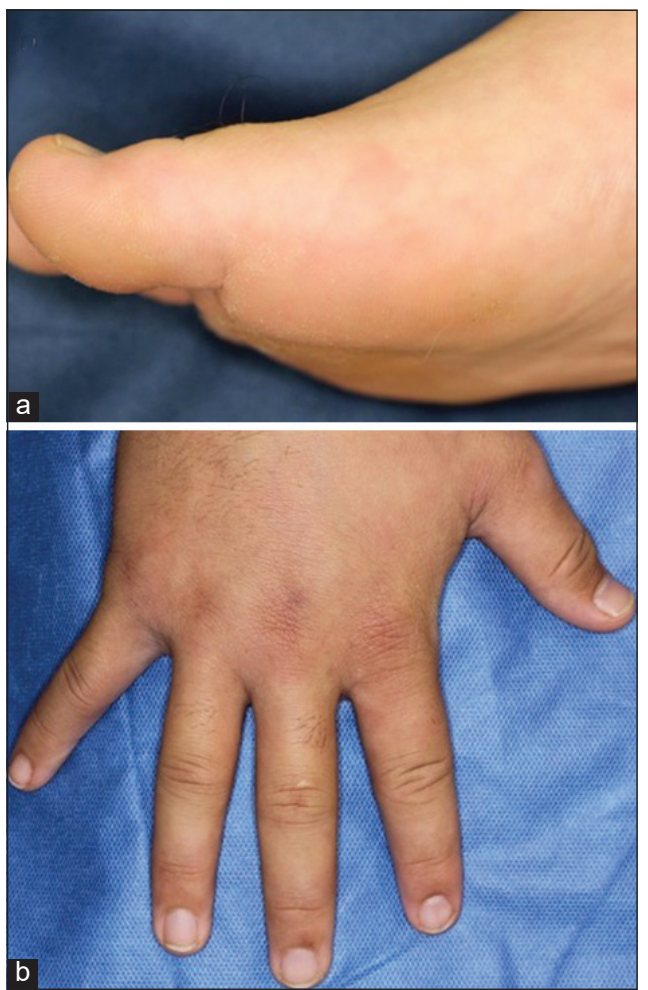

Figure 1: Salmon-pink erythemas on the feet (a), and finger swelling (b). 


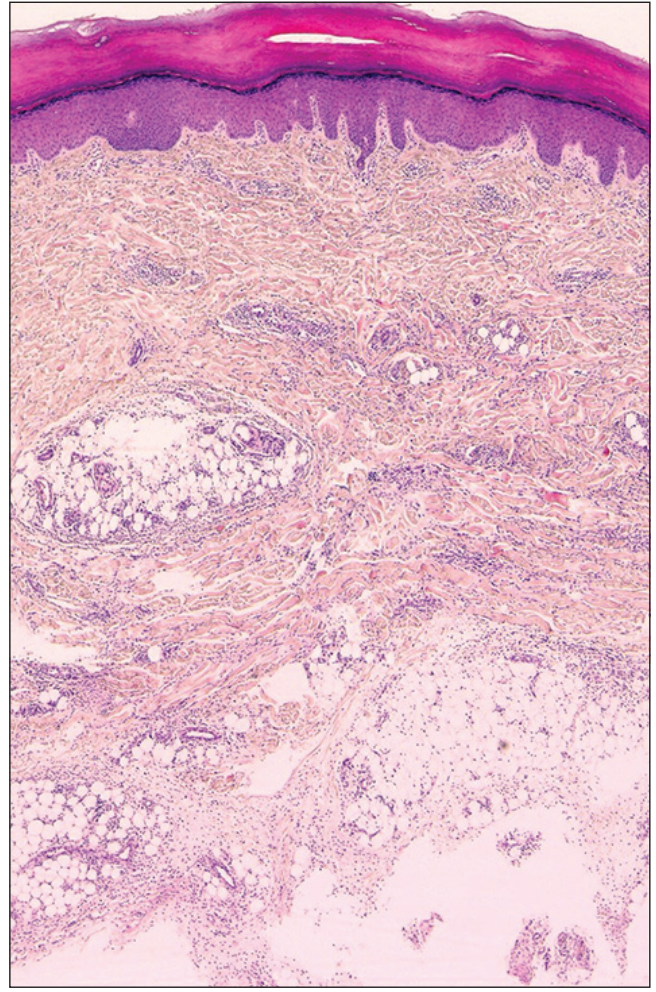

Figure 2: Histological features showing mild cellular infiltration in the dermis and subcutis $(\times 40)$.

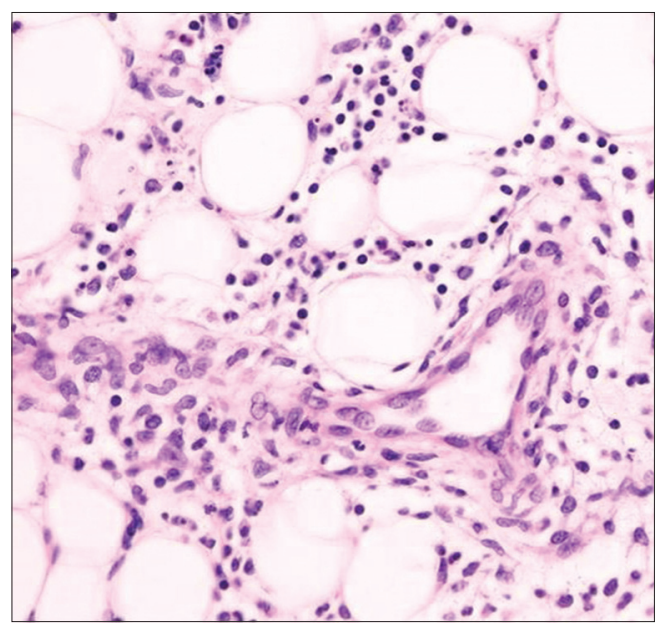

Figure 3: At higher magnification, cellular infiltrate contains mononuclear cells and neutrophils $(\times 400)$.

Sustained arthritis-related symptoms for at least 6 weeks are required for the diagnosis of sJIA, whereas AOSD patients exhibit transient arthritis/arthralgia [2]. In addition, serum ferritin levels are usually very high in patients with AOSD, but not in those with sJIA [3]. A distinct differentiation between these two diseases is sometimes difficult, and adolescent cases with this difficulty have been reported [2,4]. In the present case, the age at onset was 17, and arthralgia persisted for over 6 weeks. Serum level of ferritin was not elevated.
Thus, we diagnosed this patient as having sJIA. The patient developed recurrent erythemas, and finger swelling accompanied by fever-up. Erythema appeared in parallel with fever-up and disappeared with fever-down. Cutaneous findings such as well-circumscribed, transient, salmon-pink, macular or urticarial rashes over the trunk, face and extremities are seen in both sJIA and AOSD [5]. Histological features typically show a sparse superficial infiltration of inflammatory cells including lymphocytes and neutrophils in the upper dermis; however, the majority of histological studies have been performed mainly in AOSD, and examination in sJIA is few. In the present case, infiltration of erythema was mild, but histological examination revealed that inflammatory cells containing mononuclear cells and neutrophils were observed even in the deep dermis and subcutaneous tissues. These findings differ from those of previous reports. Furthermore, our patient developed dactylitis on both hands. Dactylitis is sometimes seen in association with various diseases such as psoriatic arthritis, tuberculosis, injury, gout, and sarcoidosis; however, the number of patients with either sJIA or AOSD presenting with dactylitis are few. The current case suggests that skin rash associated with sJIA is not only typical but also diverse.

\section{Consent}

The examination of the patient was conducted according to the Declaration of Helsinki principles.

\section{REFERENCES}

1. Fazel M, Merola JF, Kurtzman DJB. Inflammatory arthritis and crystal arthropathy: current concepts of skin and systemic manifestations. Clin Dermatol. 2018;36:533-50.

2. Prendiville JS, Tucker LB, Cabral DA, Crawford RI. A pruritic linear urticarial rash, fever, and systemic inflammatory disease in five adolescents: adult-onset Still's disease or systemic juvenile idiopathic arthritis sine arthritis? Pediatr Dermatol. 2004;21:580-8.

3. Sobieska M, Fassbender K, Aeschlimann A, Bourgeois P, Mackiewicz S, Müller W. Still's disease in children and adults: a distinct pattern of acute-phase proteins. Clin Rheumatol. 1998;17:258-60.

4. Fortna RR, Gudjonsson JE, Seidel G, DiCostanzo D, Jacobson M, Kopelman M, et al. Persistent pruritic papules and plaques: a characteristic histopathologic presentation seen in a subset of patients with adult-onset and juvenile Still's disease. J Cutan Pathol. 2010;37:932-7.

5. Chua-Aguilera CJ, Möller B, Yawalkar N. Skin manifestation of rheumatoid arthritis, juvenile idiopathic arthritis, and spondyloarthritides. Clin Rev Allerg Immunol. 2017;53:371-93.

Copyright by Takako Miura, et al. This is an open-access article distributed under the terms of the Creative Commons Attribution License, which permits unrestricted use, distribution, and reproduction in any medium, provided the original author and source are credited.

Source of Support: Nil, Conflict of Interest: None declared. 